\title{
UTILIZATION MANAGEMENT FOR EFFECTIVE HOSPITAL SERVICES
}

Riyaz A.M.M

The significant health achievements made in Sri Lanka over the last several decades need to be consolidated and further advanced given the rapidly changing demographic and epidemiological transitions seen in the country. Over the last two decades, public hospitals have undergone major expansion and re-organization without achieving all the anticipated improvements. Standardized care packages at each level and a properly functioning referral system are needed to reduce overcrowding of the specialist institutions and increase utilization of primary care units.

The fact is new medicines and diagnostic and curative technologies became available much faster than new financial resources. Therefore, no country, no matter how rich, is able to provide its entire population with every technology or intervention that may improve health or prolong life. As an example, in 2009, the British National Institute for Health and Clinical Excellence announced that the National Health Service could not offer some expensive medicines for the treatment of renal cancer because they were not cost effective (1).

Hospitals in developing countries are facing the problems of Allocative Inefficiency (resources are not cost effectively allocated), Technical Inefficiency (resources within a facility are used inefficiently), Inequity, Low Responsiveness, and poor Quality of processes. To assure that appropriate and cost effective health services are provided, health care institutions in developed countries have used Utilization Management (UM) activities for more than 20 years. UM is not only the process of managing costs and use of services through effective planning and decision-making, but also utilization management is a set of techniques used by or on behalf of purchasers of health care benefits to manage health care costs by influencing patient care decision-making through case-by-case assessments of the appropriateness of care prior to its provision.

\section{Process of Utilization Management}

Process of UM gives the answers to, practice of evidenced based medicine, cost-effectiveness and quality \& safety: Is hospitalization necessary or can surgery be done as an ambulatory procedure? How long will the patient need to be in the hospital, etc.

The process of utilization management in its most simple form involves matching patient conditions with established criteria (2), and UM is the review of services to ensure that they are medically necessary, provided in the most appropriate care setting, and at or above quality standards (3).

Utilization management is the collection, assessment and monitoring of data that pertains to patient services and treatment. UM activities evaluate many aspects of patient care, such as the timeliness of services, the number of bed days used in a hospital, the amount of medication prescribed and a patient's recovery time, so that any unnecessary services can be eliminated at hospitals. The dominant utilization management strategy is prior review of proposed medical or surgical services, which includes several related techniques such as preadmission review, continued-stay review, and second surgical opinions (4).

\section{Health care cost containment}

The rising health care costs has contributed to a growing perception that a significant amount of medical care is unnecessary and sometimes harmful. Therefore, UM has become a strong trend in health care cost containment. Under UM, some decisions are not strictly made by the doctor and patient alone. Instead, they are now checked by a reviewer reporting to an employer or other paying party who asks whether or not the proposed type or location of care is medically necessary or appropriate. Also, improving relations between hospitals and utilization reviewers may yield higher levels of utilization review compliance and significant cost savings.

\section{Components of Utilization Management in patient care}

1. Deciding who will be served:

- clarifying definitions of categories of cases to be served.

2. Assessing service needs and identifying desired outcomes:

- Timely assessments (prior to service planning).

- Thorough, appropriate, and objective 
assessments.

- Appropriate and effective tools/instruments.

- Clearly defined, measurable, meaningful, and achievable outcomes.

3. Deciding what services to provide:

- Matching services to assessed needs and desired outcomes.

- Assuring least restrictive and most cost-effective methods.

- Assuring all services are necessary and consistent with law, policy and philosophy

4. Selecting service providers and determining costs:

- Identifying the best and most appropriate providers.

5. Implementing, monitoring, changing and terminating services:

- Assuring planned services are being delivered.

- Assuring services continue to be appropriate and necessary monitoring progress toward outcomes and modifying plans as needed.

- Assuring provision and appropriate use of timely, accurate, and thorough reports thorough reports

- Assuring that transition plans are in place that are appropriate, realistic, and implemented.

Also, utilization management describes proactive procedures, including discharge planning, concurrent planning, pre-certification and clinical case appeals. Furthermore, it covers proactive processes, such as concurrent clinical reviews and peer reviews, as well as appeals introduced by the provider, payer or patient.

\section{Concurrent Review/ Discharge Planning}

Concurrent review involves screening for medical necessity and the appropriateness/ timeliness of the delivery of medical care from the time of admission until discharge. The main objectives of the concurrent review process are to ensure that doctors orders are carried out in an efficient and accurate manner, to anticipate treatment, plan ahead and to continually monitor the patient's progress and facilitate discharge planning.

Discharge planning involves preparing the patient for discharge from the facility. The process includes reviewing alternate levels of care, the need for ancillary services and the potential benefits of home support.

\section{Conclusions}

Improving health is critical to human welfare and essential to sustained economic and social development. All countries, rich and poor, struggle to raise the funds required for the health services their populations needs or demand; and are concerned to provide services of good quality cost effectively. Therefore, practicing Utilization Management will solve the problems of allocative inefficiency and low responsiveness in hospital services, and also improve the quality of services cost-effectively.

\section{Reference:}

1. NICE draft recommendation on the use of drugs for renal cancer. National Institute for Health and Clinical Excellence, 2009

(http://www.nice.org.uk/newsroom/pressreleases/p ressreleasearchive/PressReleases2009.jsp?domedia $=1 \& \mathrm{mid}=42007069$ - 19 B 9 - E 0 B 5 D429BEDD12DFE74E, accessed 3 April 2010).

2. Smith, Dean G., \& Perry, Brenda W. (1992) 'Toward Effective Hospital Utilization Management,'American Journal of Medical Quality $7: 125$.

3. Lattimer, C., \& Garrett, M. (2008). Chapter 11: Utilization management and resource management. IN CMSA Core Curriculum Case Management. Powell, S., \& Hussein, A.

4. Controlling Costs and Changing Patient Care?: The Role of Utilization Management (Free Executive Summary) http://www.nap.edu/catalog/1359.html 